SHORT REPORT

\title{
Differentiation of Parkinson's disease and atypical parkinsonian syndromes by transcranial ultrasound
}

\author{
S Behnke, D Berg, M Naumann, G Becker
}

J Neurol Neurosurg Psychiatry 2005;76:423-425. doi: 10.1136/jnnp.2004.049221

\begin{abstract}
Neuroimaging is known to complement clinical findings in the diagnostic work up of parkinsonian syndromes. Recently, transcranial ultrasound was reported to have a high diagnostic yield in differentiating idiopathic Parkinson's disease (IPD) from atypical parkinsonian syndromes. This report summarises the sonographic findings of 102 patients with IPD, 34 patients with multiple system atrophy, and 21 patients with progressive supranuclear palsy. Increased echogenicity of the substantia nigra is predictive for IPD whereas a low echogenic substantia nigra, particularly when combined with a hyperechogenic lentiform nucleus, strongly suggests an atypical Parkinsonian syndrome. The underlying causes for the differential echo pattern of the substantia nigra remain unknown.
\end{abstract}

diopathic Parkinson's disease (IPD) and atypical parkinsonian syndromes such as the parkinsonian variant of multiple system atrophy (MSA-P) and progressive supranuclear palsy (PSP) may be difficult to differentiate clinically, especially early in the disease. Nuclear medicine techniques, metabolic imaging, imaging of dopamine transporter sites or receptor density of basal ganglia nuclei, as well as routine magnetic resonance imaging (MRI) have been proposed for this purpose. However, in spite of the high technical demands and financial costs of these techniques, sensitivity and specificity are not very high. ${ }^{12}$

Recently, Walter et $a l^{3}$ proposed the use of transcranial sonography (TCS) for differentiating between IPD and atypical parkinsonian syndromes. TCS is an ultrasound based, easily applicable, low cost neuroimaging technique that allows visualisation not only of the cerebral vessels but also of brain tissue through the intact skull. Here, we describe our findings using the same technique in a larger group of patients with IPD and atypical parkinsonian syndromes.

\section{PATIENTS AND METHODS}

Two centres (the university hospitals of Homburg and Würzburg), both experienced in TCS examination, contributed their findings for this report. The study population included 102 patients with IPD ( 55 men, 47 women; median (SD) age 67 (13); median Hoehn and Yahr stage 2 (1), minimal stage 1 , maximum stage 4 ), 34 patients with MSA-P (15 men, 19 women, median age 62 (17); median Hoehn and Yahr stage 4 (1), 2-5), and 21 patients with PSP ( 16 men, five women, median age 69 (21); median Hoehn and Yahr stage 4 (1), 3-5). All the patients were white and only those patients who fulfilled the clinical diagnostic criteria for these disorders were included.

Both centres used the same technical equipment and examination protocol. TCS examination was performed through a preauricular acoustic bone window with a phased array ultrasound system, equipped with a
2.5 MHz transducer (Elegra, Siemens, Erlangen, Germany). Ultrasound system parameters were chosen as described previously (for review see reference 4). In this study we aimed to display hyperechogenic (white) areas in the basal ganglia as clearly as possible, scanning from both temporal bone windows. For the substantia nigra $(\mathrm{SN})$, the area of hyperechogenic signals in the mesencephalic scanning plane was visualised at the level of its greatest extent, encircled, and measured as described previously. ${ }^{4}$ According to the normal values of both centres, which have been obtained by examining 420 and 450 healthy adults, respectively, the extent of hyperechogenic areas in the $\mathrm{SN}$ was classified into three groups:

- low SN echogenicity: score 1 (extension of hyperechogenic signals below the 40th percentile $-0.1 \mathrm{~cm}^{2}$ and $0.11 \mathrm{~cm}^{2}$ of the healthy control groups at the two centres)

- moderate echogenicity: score 2 (40th to 80th percentile)

- marked echogenicity: score 3 (above the 80th percentile$0.18 \mathrm{~cm}^{2}$ and $0.20 \mathrm{~cm}^{2}$ at the two centres).

Moreover, echogenicity of the lentiform nucleus was rated as either normal (echogenicity same as that of the surrounding brain parenchyma) or hyperechogenic (echogenicity unequivocally elevated as compared with the surroundings). The ultrasound physicians were unaware of the clinical diagnosis of the patients.

\section{RESULTS}

A temporal bone window sufficient for an adequate sonographic analysis of the basal ganglia at least on one side was found in 88/102 patients with IPD, 32/34 patients with MSA-P, and 18/21 patients with PSP (87\% of all patients). In those patients with a sufficient temporal bone window, SNs were rated as having marked hyperechogenicity on one or both sides in $89 \%$ of the 88 patients with IPD ( $\mathrm{n}=37$ (one side) and 41 (both sides)), in $25 \%$ of the 32 patients with MSA-P ( $\mathrm{n}=2$ and 6, respectively), and in $39 \%$ of the 18 patients with PSP ( $\mathrm{n}=5$ and 2 , respectively). Moderate echogenicity of the $\mathrm{SN}$ at best on one or both sides (score 2 as the highest) was determined in 9 patients with IPD $(n=6$ (unilaterally) and 3 (bilaterally)), in 20 patients with MSA-P ( $\mathrm{n}=2$ and 18, respectively), and in 10 patients with PSP ( $\mathrm{n}=3$ and 7 , respectively). The SN was found to exhibit low echogenicity on both sides in only one patient with IPD (1\%) and with PSP $(5.5 \%)$ but in four patients with MSA-P (12.5\%) (fig 1).

Differences between the SN echogenicity of the IPD group and PSP or MSA-P group were statistically significant (Kruskal-Wallis test $\mathrm{p}<0.001$ ). Calculation of predictive

Abbreviations: IPD, idiopathic Parkinson's disease; MSA-P, parkinsonian variant of multiple system atrophy; PSP, progressive supranuclear palsy; SN, substantia nigra; TCS, transcranial sonography 


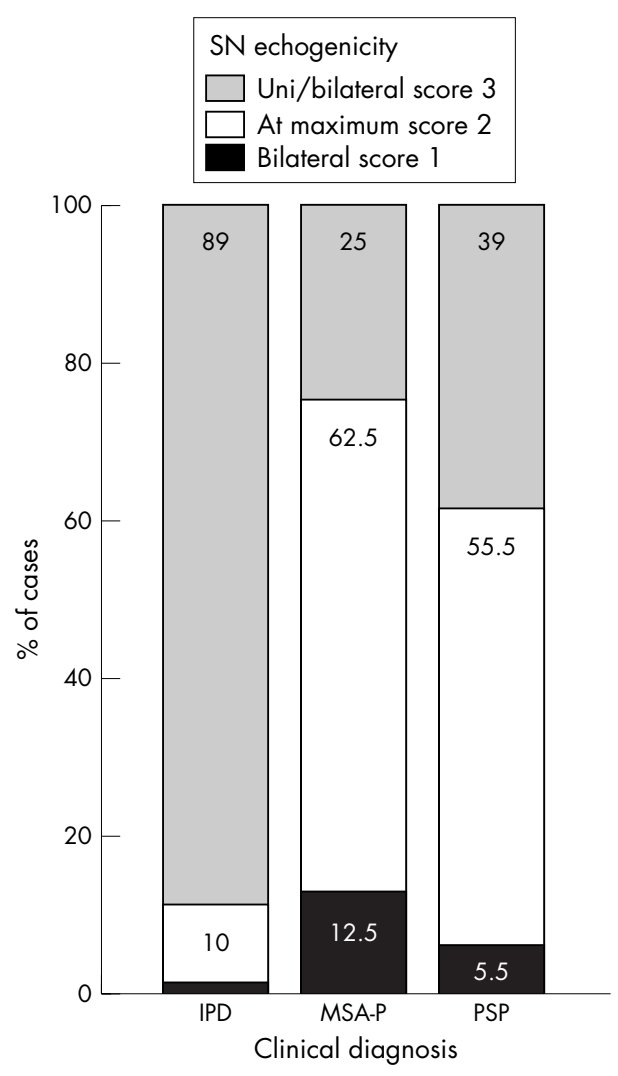

Figure 1 Percentage of cases with different ultrasound findings in the three patient groups. Grey: percentage of patients with a marked substantia nigra (SN) hyperechogenicity at least on one side; black: percentage of patients with a normal brainstem signal bilaterally; and white: percentage of patients who exhibited a moderate increase in SN echogenicity unilaterally or bilaterally but no signal extent above the 80th percentile. IPD, idiopathic Parkinson's disease; MSA-P, parkinsonian variant of multiple system atrophy; PSP, progressive supranuclear palsy.

values revealed that, in a patient with parkinsonian syndrome, the sonographic finding of a distinctly hyperechogenic SN on one or both sides predicted IPD with positive and negative predictive values of 0.83 and 0.78 , respectively.

There were no statistically significant correlations between disease severity within the IPD group (Hoehn and Yahr stage) and extent of the hyperechogenic SN signal (Pearson's and Spearman's correlation coefficients).

Whereas unilateral or bilateral hyperechogenicity of the lentiform nucleus was found in 13/18 patients with PSP and in 23/32 patients with MSA-P, only 10 patients with IPD showed this echo feature. Differences between these groups were significant (Kruskal-Wallis test $\mathrm{p}<0.01$ ). Hyperechogenic lentiform nuclei as an indicator for an atypical parkinsonian syndrome had positive and negative predictive values of 0.78 and 0.84 , respectively. When both ultrasound variables were combined, the positive predictive value of ultrasound in identifying IPD and atypical parkinsonian syndromes increased significantly (table 1).

\section{DISCUSSION}

In the present study, in most patients with IPD (88\%) a markedly hyperechogenic SN was detected on at least one side corroborating the results of previous studies. ${ }^{45}$ In contrast, a hyperechogenic lentiform nucleus was more frequent in the atypical parkinsonian syndromes. Thus, patients with "parkinsonism" who exhibit only a low echogenic SN-particularly when combined with a hyperechogenic lentiform nucleus-are likely to have an atypical parkinsonian syndrome (positive predictive value 0.96), whereas a significantly hyperechogenic SN points towards IPD (positive predictive value 0.91 , see table 1 ). Although this confirms the findings of Walter et al, ${ }^{3}$ the present positive and negative predictive values are somewhat lower than those reported in their study.

The ultrasound finding of a hyperechogenic lentiform nucleus corresponds to a decrease in $\mathrm{T} 2$ relaxation time on MRI, ${ }^{6}$ which is supposed to be related to an increase in the heavy metal concentration in this area.

The underlying reason for the differences in the pattern of SN echogenicity of IPD and atypical PD is unknown. Evidence from animal ${ }^{7}$ and post mortem ${ }^{8}$ studies points to a relation between SN echogenicity and tissue iron content. However, tissue iron has been found to be elevated not only in IPD but also in MSA-P and PSP. ${ }^{9}$ In vitro studies by our group have shown that iron itself does not cause an increase in ultrasound signal intensity (unpublished data). However, it has been hypothesised that binding of increased amounts of iron to iron metabolising proteins or structural alteration of iron binding proteins might lead to differences in the reflection of the ultrasound beam displayed as hyperechogenic signals. This hypothesis was substantiated by a recent study on association of an increase of the iron binding proteins ferritin-H and ferritin-L and SN hyperechogenicity. ${ }^{10}$ Moreover, it has been shown that sequence variations in genes encoding for iron metabolising proteins can be linked to IPD $^{11}{ }^{12}$ and hyperechogenicity of the SN. ${ }^{12}$ The kinds of difference between iron metabolism in IPD and in atypical parkinsonian syndromes therefore need to be further elucidated. Additionally, it remains to be investigated what other factors might be involved in the increase of SN echogenicity in IPD.

In the past, use of other imaging tools has been proposed in the differential diagnosis of parkinsonian syndromes. Several positron emission tomography (PET), single photon emission

Table 1 Number of patients with two distinct ultrasound findings: one combining a hyperechogenic substantia nigra (SN) on at least one side with a regular echogenic lentiform nucleus (LN) on both sides and the other combining a hyperechogenic LN on one or both sides with a moderate or low echogenic SN. The first combination together with the clinical finding of "parkinsonism" reliably predicted IPD, whereas the second combination was indicative of an atypical parkinsonian syndrome

\begin{tabular}{|c|c|c|c|}
\hline \multirow[b]{2}{*}{ Echo pattern of the $S N$ and the $L N$} & \multicolumn{2}{|l|}{ No of patients } & \multirow[b]{2}{*}{ Positive predictive value } \\
\hline & With Parkinson's disease & With atypical parkinsonian syndromes & \\
\hline $\begin{array}{l}\text { Hyperechogenic } \mathrm{SN} \text { combined with } \\
\text { regular echogenic } \mathrm{LN}\end{array}$ & 58 & 6 & 0.91 \\
\hline $\begin{array}{l}\text { Hyperechogenic } L N \text { combined with } \\
\text { moderate echogenic } S N \text { at best }\end{array}$ & 1 & 28 & 0.96 \\
\hline
\end{tabular}


computed tomography (SPECT), and MRI variables have been identified as being different in IPD, MSA-P, and PSP. ${ }^{12}$ However, most of the findings overlap and provide only moderate accuracy with respect to differential diagnosis of these entities, thus failing to be of significant use for a given patient contact. In addition, these procedures are time consuming, costly, and have limited availability. Recently, MRI volumetry and diffusion-weighted imaging based MRI has been introduced which allows discrimination of atypical PD from IPD with high sensitivity and specificity. ${ }^{13}{ }^{14}$ Since none of these tools, ultrasound included, visualises a unique pathogenetic feature of any of these disorders, it remains questionable whether these imaging tools can be more than a valuable support to the clinical diagnosis. Each imaging procedure will have to be embedded into the clinical context. Therefore, it is of relevance whether the imaging tool is noninvasive, easily available, and applicable by the clinician. These are the immanent advantages of diagnostic ultrasound imaging, which may outweigh the drawback of investigator dependency in the future.

\section{Authors' affiliations}

S Behnke, G Becker, Department of Neurology, University of Homburg, Saar, Germany

D Berg, Hertie Institute for Clinical Brain Research and Institute for Medical Genetics, University of Tuebingen, Germany

M Naumann, G Becker, Department of Neurology, University of Würzburg, Germany

This study was supported by grants from the Kompetenznetzwerk Parkinson, Germany, and the Siemens AG.

Competing interests: none declared

Correspondence to: Dr S Behnke, Department of Neurology, University of the Saarland, Gebäude 9066421 Homburg/Saar, Germany; nesbeh@uniklinik-saarland.de
Received 5 July 2004

Accepted 2 August 2004

\section{REFERENCES}

1 Schrag A, Good CD, Miszkiel K, et al. Differentiation of atypical parkinsonian syndromes with routine MRI. Neurology 2000;54:697-702.

2 Brucke T, Djamshidian S, Bencsits G, et al. SPECT and PET imaging of the dopaminergic system in Parkinson's disease. J Neurol 2000;247(suppl 4):IV/2-7.

3 Walter U, Niehaus L, Probst T, et al. Brain parenchyma sonography discriminates Parkinson's disease and atypical parkinsonian syndromes. Neurology 2003;60:74-7.

4 Berg D, Becker G. Perspectives of B-mode transcranial ultrasound. Neurolmage 2002;15:463-73.

5 Walter $U$, Wittstock $M$, Benecke $R$, et al. Substantia nigra echogenicity is normal in non-extrapyramidal cerebral disorders but increased in Parkinson's disease. J Neural Transm 2002;109:191-6.

6 Berg D, Naumann M, Hoggenmuller U, et al. Differences of echogenicity and T2 relaxation time of the nucleus lentiformis in MSA and PSP as compared to Parkinson's disease. Mov Disord 2000;15:206.

7 Berg D, Roggendorf W, Schroder U, et al. Echogenicity of the substantia nigra: association with increased iron content and marker for susceptibility to nigrostriatal injury. Arch Neurol 2002;59:999-1005.

8 Berg D, Grote C, Rausch WD, et al. Iron accumulation of the substantia nigra in rats visualized by ultrasound. Ultrasound Med Biol 1999;25:901-4.

9 Dexter DT, Jenner P, Schapira AH, et al. Alterations in levels of iron, ferritin, and other trace metals in neurodegenerative diseases affecting the basal ganglia. The Royal Kings and Queens Parkinson's Disease Research Group. Ann Neurol 1992;32(suppl):S94-100.

10 Zecca L, Berg D, Becker G, et al. The in vivo detection of iron and ferritins by transcranial sonography: a new approach for early diagnosis of Parkinson's disease. Mov Disord 2002;17(suppl 5):S181.

11 Borie C, Gasparini F, Verpillat P, et al. Association study between iron-related genes polymorphisms and Parkinson's disease. J Neurol 2002;249:801-4.

12 Hochstrasser H, Felletschin B, Schöbel K, et al. Sequence variations in genes involved in iron metabolism in Parkinson's disease. Medgen 2003; 15:240.

13 Seppi K, Schocke MFH, Esterhammer R, et al. Diffusion-weighted imaging discriminates progressive supranuclear palsy from PD, but not from the Parkinson variant of multiple system atrophy. Neurology 2003;60:922-7.

14 Schulz JB, Skalej M, Wedekind D, et al. Magnetic resonance imaging-based volumetry differentiates idiopathic Parkinson's syndrome from multiple system atrophy and progressive supranuclear palsy. Ann Neurol 1999;45:65-74. 